\title{
Vasoconstrictive responses of the cephalic vein during first-time cardiac implantable electronic device placement
}

\author{
R. Steckiewicz, E.B. Świętoń, M. Bogdańska, P. Stolarz \\ Department of Cardiology, Medical University of Warsaw, Poland \\ [Received: 20 September 2017; Accepted: 14 December 2017]
}

Background: During cardiac implantable electronic device (CIED) implantation procedures cardiac leads have been mostly introduced transvenously. The associated injury to the selected vessel and adjacent tissues may induce reflex vasoconstriction. The aim of the study was to assess the incidence of cephalic vein (CV) vasoconstriction during first-time CIED implantation.

Materials and methods: Of the 146 evaluated first-time CIED implantation procedures conducted in our centre in 2016, we selected those during which $C V$ vasoconstriction was recorded. We focused on the stage of the procedure involving CV cutdown and/or axillary vein (AV)/subclavian vein (SV) puncture for lead insertion. Only cases documented via venography were considered.

Results: Vasoconstriction was observed in 11 patients ( 5 females and 6 males, mean age $59.0 \pm 21.2$ years). The presence of this phenomenon affected the stage of CIED implantation involving cardiac lead insertion to the venous system, in severe cases, requiring a change of approach from CV cutdown to AVISV puncture. The extent of vasoconstriction front propagation was limited to the nearest valves. Histological examinations of collected CV samples revealed an altered spatial arrangement of myocytes in the tunica media at the level of leaflet attachment. Conclusions: Cephalic vein vasoconstriction is a rare phenomenon associated with accessing the venous system during first-time CIED implantation. The propagation of CV constriction was limited by the location of the nearest valves. (Folia Morphol 2018; 77, 3: 464-470)

Key words: cephalic vein, venous spasm, tunica media, venotomy, puncture, lead implantation, cardiac implantable electronic device

\section{INTRODUCTION}

A vast majority of currently performed cardiac implantable electronic device (CIED) implantation procedures use transvenous cardiac lead placement. This is performed by one of two techniques: cephalic vein (CV) cutdown and/or either axillary vein (AV) or subclavian vein (SV) puncture. All these vessels are typically accessed in the clavipectoral triangle [1, 7].
Using CV cutdown is associated with less potential for damaging the structures surrounding the target vessel, which is why this technique is typically the firstline method in cardiac lead placement. One common obstacle in cardiac lead insertion via CV cutdown and further lead advancement along the CV are the vessel's anatomical variations in the form of its atypical structure and/or location. This obstacle has led to

Address for correspondence: R. Steckiewicz, MD, PhD, Department of Cardiology, Medical University of Warsaw, ul. Banacha 1A, 02-097 Warszawa, Poland, tel: +48 22599 2958, e-mail: r.steckiewicz@pro.onet.pl 
improvements in the technique of lead insertion and advancement via the CV $[6,8,12]$. However, there is another obstacle that may also hinder transvenous lead insertion, and that is reflex vasoconstriction. This aspect of CIED implantation procedures has been mainly reported with respect to large veins $[2,3,11]$.

First-time CIED implantation with transvenous lead placement is associated with various forms of injury to target vessels and the surrounding tissues. This injury, depending on its nature and extent, may induce an increase or decrease in vascular wall tension, leading to venous vasoconstriction or vasodilation, respectively. The extent and dynamics of these reactions are closely associated with venous wall composition, with the tunica media being the layer mostly responsible for this reaction, due to its myocyte content.

From an anatomical point of view the $\mathrm{CV}$ is classified as a superficial vein; unlike some other veins, this one has no corresponding artery. From a histological point of view $\mathrm{CV}$ is classified as a medium vein; these can vary in terms of their lumen diameter, wall thickness, and the presence of valves controlling the direction of blood flow. Histological characteristics of the layers, including the tunica media, that from CV walls may change in patients with severe kidney failure [13].

The aim of this study was to analyse the phenomenon of reflex functional $\mathrm{CV}$ vasoconstriction during procedures of first-time CIED implantation and to illustrate it using images acquired as part of our practice. We assessed the vasoconstrictive reaction within the segment of the vessel lying within the clavipectoral triangle, which is the area used for cardiac lead insertion via the CV cutdown technique. To the authors' best knowledge, this aspect of CIED implantation has not been reported in other papers.

\section{MATERIALS AND METHODS}

A total of 377 first-time transvenous CIED implantation procedures were conducted in our centre in 2016. For our analysis we selected 146 procedures that were conducted by the same operator. This criterion eliminated such variables as operator's experience, lead insertion technique, and differences in subjective interpretation of the evaluated phenomenon. $\mathrm{CV}$ vasoconstriction was evaluated only in the vascular segment situated in the clavipectoral triangle, which was used for first-time CIED implantation.
The procedures involved three types of lead insertion: CV cutdown only, CV cutdown and AV/SV puncture to insert an additional lead, and AV/SV puncture after an unsuccessful attempt at lead insertion via CV cutdown. In every case where both techniques were used, it was always CV cutdown that was performed first.

We assessed vasoconstriction-induced CV narrowing/occlusion manifesting via reduced/ceased blood flow in the evaluated vascular segment. Any vasoconstriction (reduced venous diameter) observed during venography or via vessel exposure in the clavipectoral groove has been documented with captured images. This allowed subsequent exclusion of cases that were inconclusive, questionable, or not directly related to the topic of the study (e.g. the presence of local residual thrombotic lesions). The images presented in this paper illustrate non-repetitive, unique cases.

The contrast media used throughout the study were nonionic low-osmolality monomers (Iomeron). The flow of contrast through the $\mathrm{CV}$ was achieved by administering a $15-30-\mathrm{mL}$ volume (mean $20 \mathrm{~mL}$ ) of contrast, with the variation resulting from interindividual variations in venous bed volume resulting from the selected contrast administration site.

Contrast medium flow was recorded via a GE OEC 9900 Elite workstation (anteroposterior view). Pulse fluoroscopy of $8 \mathrm{Fr} / \mathrm{s}$ was used for the purpose of both conducting the procedures at hand as well as documenting them. Venous vasoconstriction was assessed based on changes in the vessel's width, as determined by contrast flow captured in individual frames.

In selected cases 2-4-mm CV segments were collected at the final stage of the procedure. The tissue samples were fixed in a $4 \%$ buffered formalin solution, processed into paraffin blocks, cut into 4-micron-thick sections, deparaffinised, and, subsequently, stained with: haematoxylin and eosin, Masson's trichrome stain for smooth muscle actin. The evaluated cases were those with normal chemical parameters of kidney function and normal glomerular filtration rate. The histological sections of CV presented in Figure 1.

This study had been approved by the Institutional Review Board at the medical centre.

Statistical variables were expressed as means and standard deviations.

\section{RESULTS}

A total of 146 (38.7\%) cases, out of the 377 firsttime CIED implantation procedures performed in 


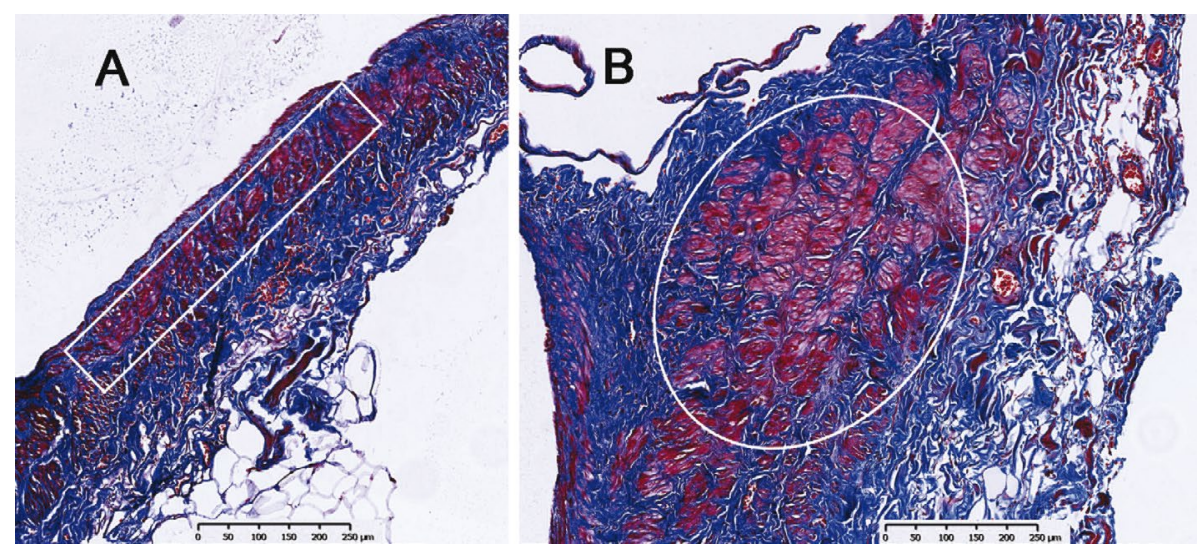

Figure 1. Histological slide containing longitudinal sections of cephalic vein segments: the segment upstream of the valve $(\mathbf{A})$ and at the valve base (B). Masson's trichrome stain visualises alternating collagen fibres (in blue) and muscle fibres (in red) in the tunica media (magnification $\times 100$ ). A cardiac implantable electronic device implantation procedure with no apparent reflex vasoconstriction; A. Vascular wall fragment that illustrates muscular layer structure (rectangle); B. The base of a valve, with local venous wall thickening (oval), due mainly to increased numbers or size (i.e. hypertrophy) of muscle cells.
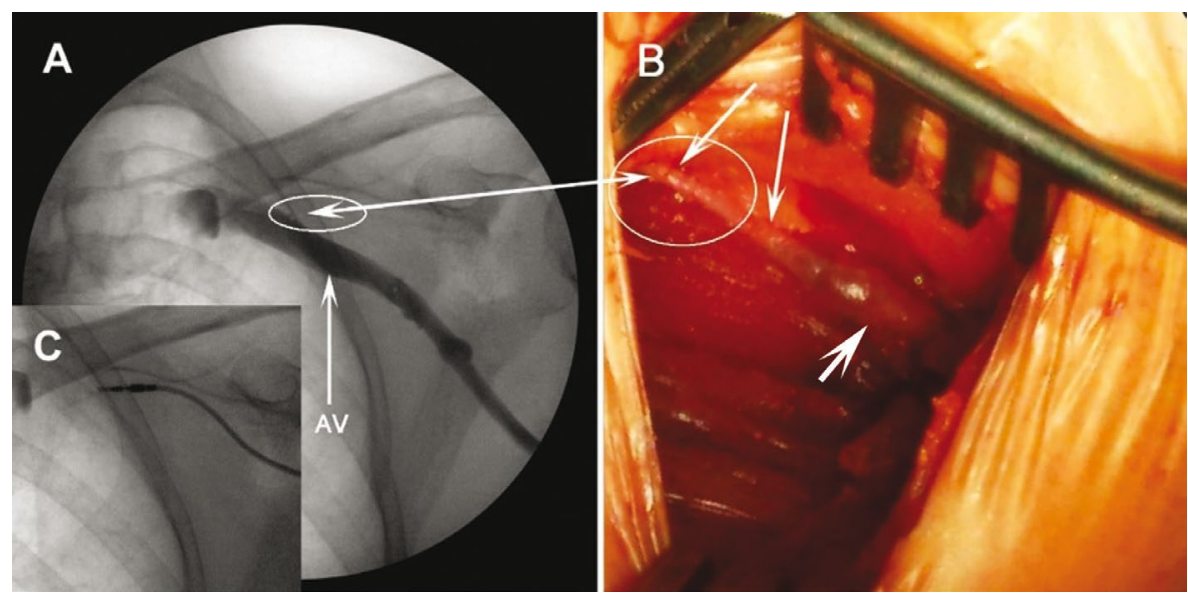

Figure 2. A. Venography in a 71-year-old woman; the complete lack of contrast flow at the typical anatomical location of cephalic vein (CV) (double-headed arrow + oval) suggests the absence of the vessel; B. Macroscopic view of the cause for lack of contrast flow from panel A: a segmental vasoconstriction of CV (arrows + oval) with the vasodilation beginning peripherally (wide arrow) and moving more centrally; C. A lead advanced through CV lumen after the vasoconstriction subsided confirmed CV patency; AV — axillary vein.

2016, were selected according to the adopted criteria. The selected study group comprised 68 females (mean age $77 \pm 13$ years) and 78 males (mean age $73 \pm 13$ years); 115 patients (mean age $78.5 \pm 10.1$ years) received pacemakers (PM), while 31 patients (mean age $62.5 \pm 15.9$ years) received cardioverter-defibrillators. In $80(54.8 \%)$ cases (mean age $75.1 \pm 11.7$ years) cardiac lead placement was conducted via CV cutdown only; in $10(6.9 \%)$ cases (mean age $82.9 \pm 6.9$ years) $\mathrm{CV}$ cutdown was used in conjunction with AV/SV puncture. These two subgroups constituted $62 \%$ of procedures in the evaluated group. In the remaining $56(38 \%)$ cases the leads were introduced into the venous system via $\mathrm{AV} / \mathrm{SV}$ puncture only.
The evaluated phenomenon, captured in venography images, occurred in 11 patients ( 5 females and 6 males; mean age $59.0 \pm 21.2$ years). Figures $2-7$ present distinctive cases.

In $6(5 \%)$ cases, the degree of CV segmental vasoconstriction required AV/SV puncture to accomplish cardiac leads placement. In 3 cases, the leads were advanced beyond the constricted $\mathrm{CV}$ segment after a guidewire was passed through, without the need for AV/CV puncture. In 2 cases it was possible to perform another, this time successful, attempt at lead advancement, due to the gradual, spontaneous resolution of CV vasoconstriction. 

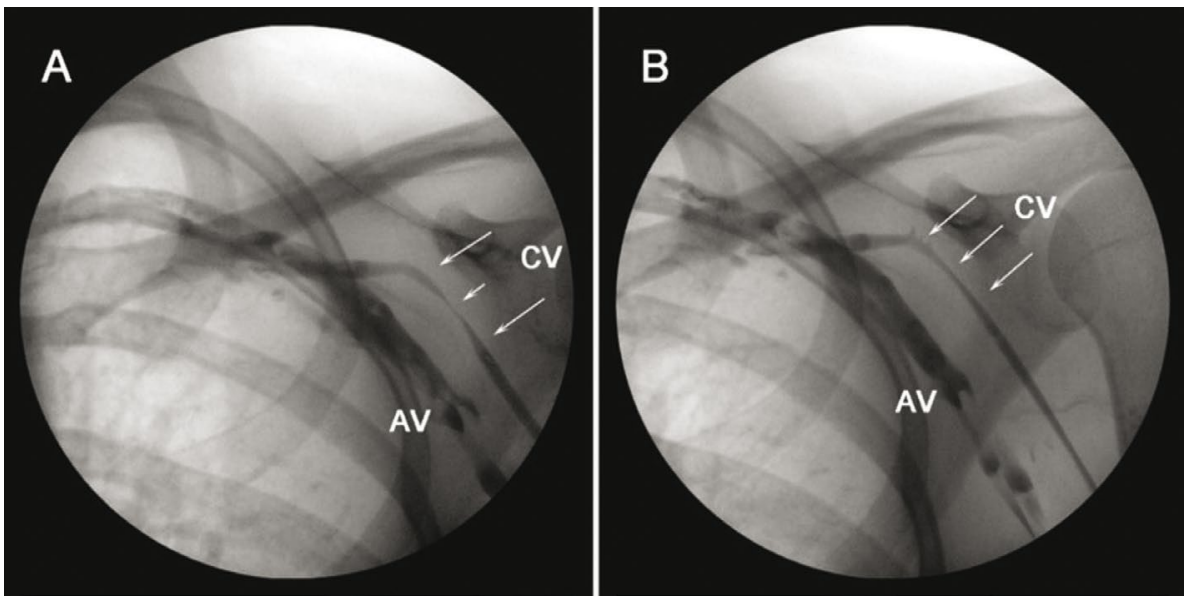

Figure 3. An 18-year-old female; A. Segmental cephalic vein (CV) vasoconstriction narrowing the lumen and limiting the flow of contrast (short arrow), with a peripheral dilation of the vessel caused by inflowing and accumulating contrast medium (long arrows); B. Follow-up venography illustrated a gradual normalisation of contrast flow; AV — axillary vein.
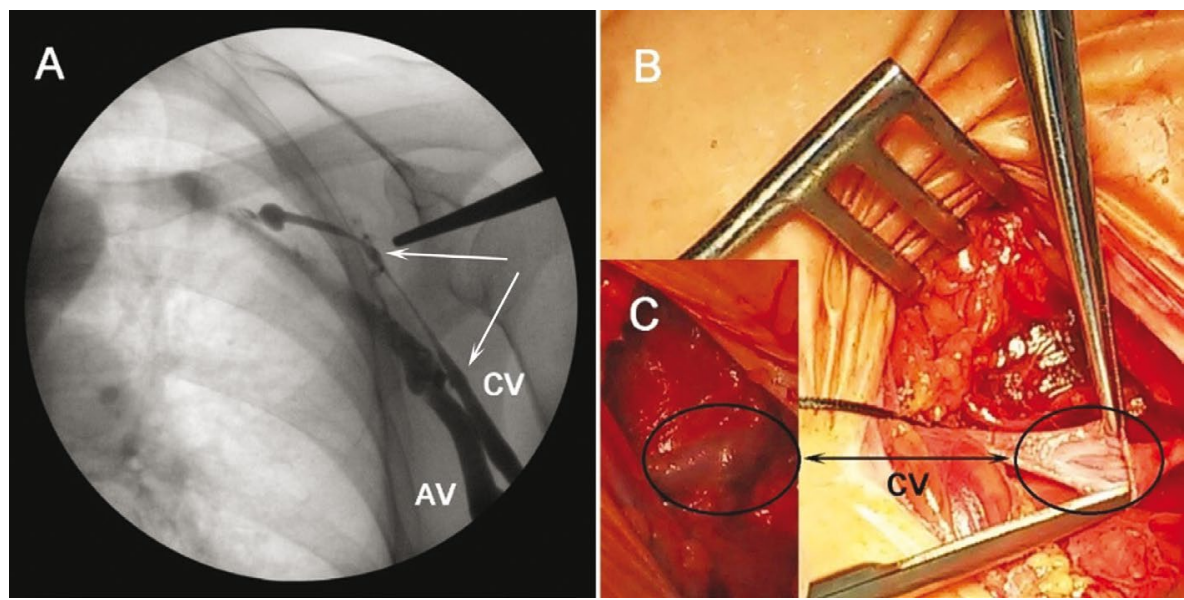

Figure 4. A 59-year-old female; A. Segmental cephalic vein (CV) vasoconstriction between the arrowheads (white arrows); B, C. A valve (black oval) — indicated with a tool in panels A and B; view before (panel C) and after (panel B) vein transection; B. Valve leaflets; C. Venous walls moulded on valve leaflet attachment lines; AV — axillary vein.
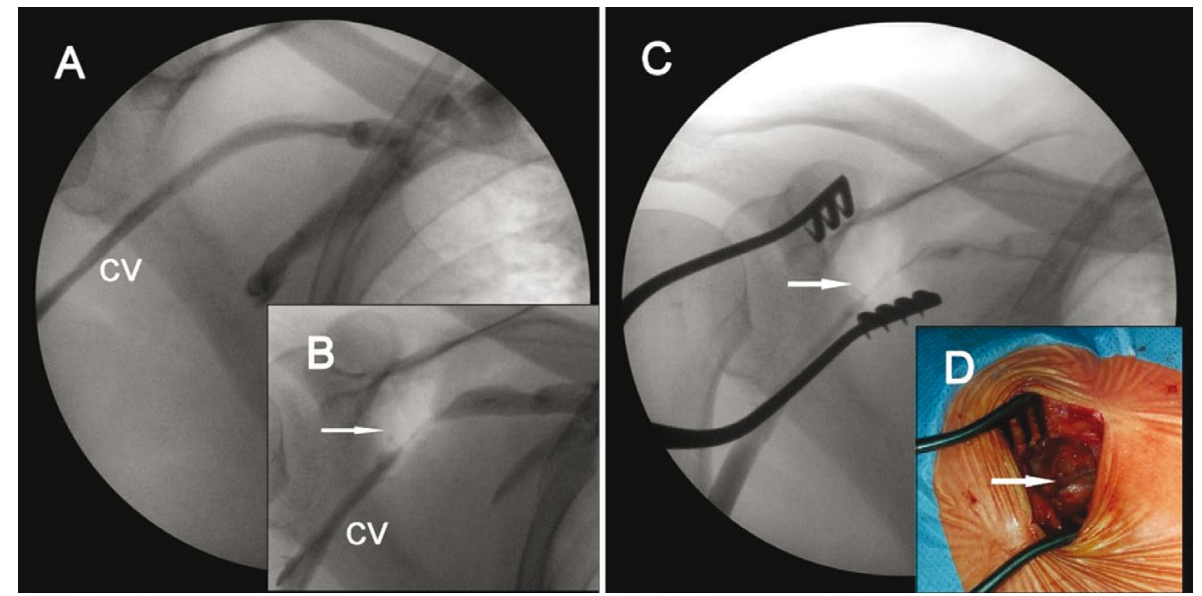

Figure 5. A 42-year-old male; A. A venography image depicting a cephalic vein (CV) whose course was favourable for lead insertion with the cutdown technique; B. Segmental CV vasoconstriction, which occurred at the beginning of vein dissection (arrow); C, D. CV vasoconstriction, persisting after the vessel was fully exposed (arrow). A thinning in the layer of tissues above the vessel (panel D) lowers the local intensity of the background of the image (panels $B, C$ ). 

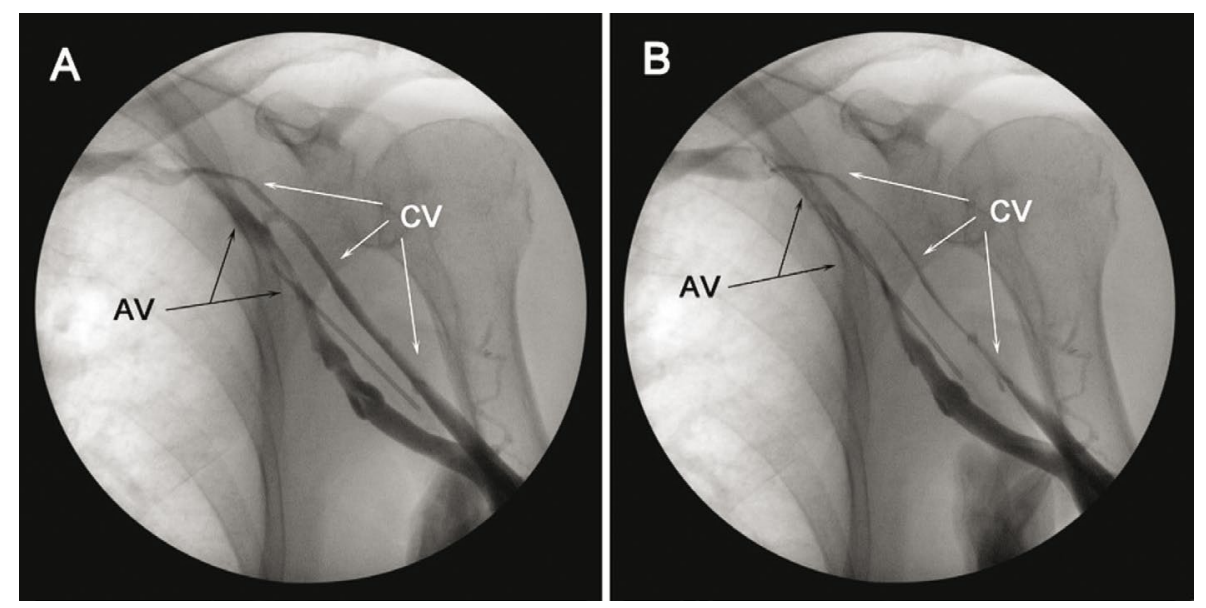

Figure 6. A 74-year-old female; cephalic vein (CV) vasoconstriction induced by axillary vein (AV) puncture; A. Insertion of a needle into the AV (long black arrow) induced constriction of the punctured vessel (black arrows) and a similar constriction of CV; B. The segment of CV vasoconstriction reaction shifted peripherally (white arrows).
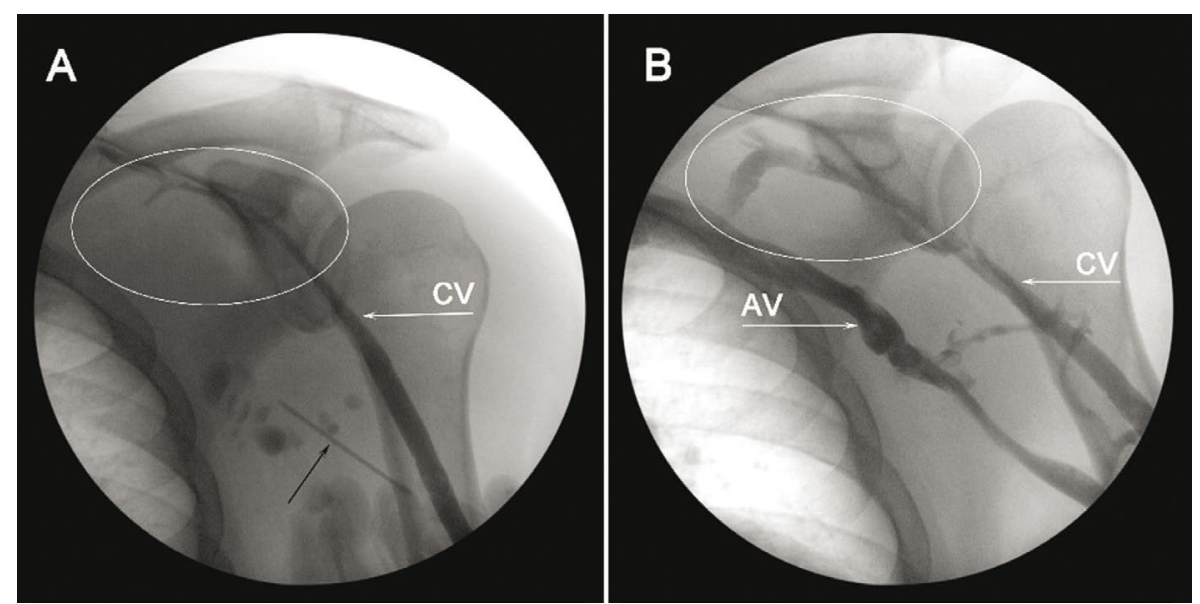

Figure 7. A 69-year-old male; A. A cephalic vein (CV) characterised by unfavourable morphometry for lead insertion (oval). An attempt at axillary vein (AV) puncture (black arrow); no visible flow of contrast in the $\mathrm{AV}$, with contrast accumulation at a venous valve; $B$. Venographic image obtained 2 days later showed complete patency of the AV and typical CV morphometry. The diameter of the vessel afforded insertion of both leads of a DDD pacing system (oval).

In the authors' judgment there were 2 cases of CV vasodilation, with the walls of the vessel becoming flaccid to a degree that caused lumen closure and blood flow cessation. However, due to the acquired images being inconclusive, this phenomenon was not further analysed in this paper.

In the remaining 133 (91\%) cases there were no definitive or readily documentable changes in the lumen diameter in the evaluated $\mathrm{CV}$ segment.

Tissue injury associated with CV dissection or AV/ ISV puncture, in some cases, induced vasoconstriction in the CV segment lying in the clavipectoral triangle. The narrowness of the vessel, revealed after it was dissected free, suggested the vessel's natural anatomical structure unfavourable for lead insertion. This finding supported AV/SV puncture as the preferred approach in patients with no prior venography to demonstrate the actual CV morphometry (situation from Fig. 7).

Except for the vascular segment at the base of a valve, the histological structure of the CV was characterised by circularly arranged muscle fibres within the tunica media, with no changes in tunica media thickness (Fig. 1A). The presence of a valve was characterised by vascular wall thickening at the valve base. This local change in venous morphology was due to an increased number of myocytes and/ /or myocyte hypertrophy. At valve bases, apart from 
circular muscle fibres, there were also longitudinal strands of myocytes (Fig. 1B).

\section{DISCUSSION}

Morphometric parameters and anatomical course of three vessels: the $C V, A V$, and $S V$, determines the effectiveness of the first stage of CIED implantation procedures involving transvenous cardiac lead placement. The peripheral segment of $\mathrm{CV}$ courses along the lateral aspect of the forearm and arm, with some of its segments visible underneath the skin (at the level of the wrist and antecubital fossa). The visible venous segments are typically used during simple invasive procedures, such as drawing blood and administering intravenous drugs, whereas the deeper, proximal CV segment lying in the clavipectoral groove has been used during more complex interventions, such as venous access port placement and CIED implantation procedures.

Cardiac lead insertion and passage through these vessels may be hindered by congenital factors, such as anatomical variations in vascular parameters and position or acquired, organic factors, such as lumen obstruction due to local residual thrombotic lesions following an inflammatory process (induced by injections, indwelling vascular-access devices, etc.) $[5,10]$.

Vascular patency can be also affected by reactive vasoconstriction, sometimes to the extent that hinders lead advancement. Reactive constriction or relaxation of vessel wall muscles is determined by the intensity of stimuli received by autonomic nervous system receptors and by local metabolic factors. The resulting manifestations depend on the diameter of vascular lumen, length of the closed vascular segment, and duration of blood flow obstruction.

Vasoconstrictive reactions, involving the $\mathrm{CV}$ segment lying in the clavipectoral triangle, sometimes occur during CIED implantation procedures. Discovering that the $\mathrm{CV}$ does not favour lead advancement requires a change in lead insertion technique from $\mathrm{CV}$ cutdown to AV/SV puncture, a technique with a higher risk of intraprocedural complications, such as pneumothorax or arterial puncture, and delayed sequelae, such as crush syndrome [4].

Vasoconstriction, due to activated circumferential muscular fibres of the tunica media, may be induced by temperature changes, chemical factors (such as medication, contrast media), indirect (compression) or direct (puncture, catheter insertion) application of mechanical forces to the adjacent tissues $[2,11]$.
$\mathrm{CV}$ vasoconstriction observed in our study group was induced by handling the affected vessel segment itself (tissue dissection to expose the vessel) and by injuring the wall of a nearby vessel (AV/SV puncture). The extent of vasoconstriction was typically determined by the location of $\mathrm{CV}$ valves, if any were present in the evaluated segment. One conceivable explanation for this finding are the observed histological differences in tunica media myocyte arrangement at valve sites.

During the study, we encountered isolated cases of $\mathrm{CV}$ vasodilation, with lumen collapse. However, in these cases we cannot exclude a vasodilatory effect of lidocaine (used for local anaesthesia) combined with inadequate patient hydration (due to a reduced amount of fluids ingested prior to the procedure) [9].

The lack of available papers on $\mathrm{CV}$ vasoconstriction precluded drawing any comparisons with respect to our findings. The only available reports mentioning vasoconstriction in first-time transvenous CIED implantation procedures are either isolated case reports or papers describing constriction of larger-calibre vessels $[2,3]$.

\section{Limitations of the study}

The normal CV diameter in some cases can be relatively small, which might confound an objective assessment of vasoconstriction. In order to avoid this type of uncertainty, our assessment included only the cases of vasoconstriction that could be clearly documented via imaging.

In the study group we (relatively more frequently) encountered cases, where the manoeuvrability of an already inserted lead or an insertion of another lead were hindered, seemingly due to CV walls constricting around an already inserted lead. However, due to the subjective nature of these findings and difficulties in documenting these cases objectively, we decided against including these cases in the present report.

The restrictions presented above limited the number of evaluated (reportable) procedures in our study. Nonetheless, we believe the evaluated phenomenon to have been documented in a sufficiently objective manner for our findings to be extrapolated onto larger populations of this type of patients.

\section{CONCLUSIONS}

Cephalic vein vasoconstriction during first-time CIED implantation procedures is a rare phenomenon; however, in some cases the degree of constriction 
affects the course of the procedure, e.g. requires changing lead insertion technique from $\mathrm{CV}$ cutdown to $\mathrm{AV} / \mathrm{SV}$ puncture.

The propagation of $\mathrm{CV}$ vasoconstriction along the vessel in the evaluated cases was limited by the presence of the nearest venous valves.

\section{REFERENCES}

1. Bongiorni MG, Proclemer A, Dobreanu D, et al. Preferred tools and techniques for implantation of cardiac electronic devices in Europe: results of the European Heart Rhythm Association survey. Europace. 2013; 15(11): 1664-1668, doi: 10.1093/europace/eut345, indexed in Pubmed: 24170423.

2. Cooper RM, Krishnan U, Pyatt JR. Central venous spasm during pacemaker insertion. Heart. 2010; 96(18): 1484, doi: 10.1136/hrt.2010.203919, indexed in Pubmed: 20813728.

3. Duan $\mathrm{Xu}$, Ling $\mathrm{F}$, Shen $\mathrm{Y}$, et al. Venous spasm during contrast-guided axillary vein puncture for pacemaker or defibrillator lead implantation. Europace. 2012; 14(7): 1008-1011, doi: 10.1093/europace/eus066, indexed in Pubmed: 22436615.

4. Jacobs DM, Fink AS, Miller RP, et al. Anatomical and morphological evaluation of pacemaker lead compression. Pacing Clin Electrophysiol. 1993; 16(3 Pt 1): 434-444, indexed in Pubmed: 7681195.

5. Kodani T, Mine T, Kishima H, et al. Spontaneous subclavian venous occlusion before electronic device implantation. Asian Cardiovasc Thorac Ann. 2015; 23(5): 530-534, doi: 10.1177/0218492314567925, indexed in Pubmed: 25614481.
6. Kolettis TM, Lysitsas DN, Apostolidis D, et al. Improved 'cutdown' technique for transvenous pacemaker lead implantation. Europace. 2010; 12(9): 1282-1285, doi: 10.1093/ europace/euq173, indexed in Pubmed: 20519193.

7. Loukas M, Myers CS, Wartmann ChT, et al. The clinical anatomy of the cephalic vein in the deltopectoral triangle. Folia Morphol. 2008; 67(1): 72-77, indexed in Pubmed: 18335417.

8. Neri R, Cesario AS, Baragli D, et al. Permanent pacing lead insertion through the cephalic vein using an hydrophilic guidewire. Pacing Clin Electrophysiol. 2003; 26(12): 2313-2314, indexed in Pubmed: 14675018.

9. Newton DJ, McLeod GA, Khan F, et al. Mechanisms influencing the vasoactive effects of lidocaine in human skin. Anaesthesia. 2007; 62(2): 146-150, doi: 10.1111/j.13652044.2006.04901.x, indexed in Pubmed: 17223807.

10. Oginosawa $Y$, Abe $H$, Nakashima $Y$. Prevalence of venous anatomic variants and occlusion among patients undergoing implantation of transvenous leads. Pacing Clin Electrophysiol. 2005; 28(5): 425-428, doi: 10.1111/j.15408159.2005.09534.x, indexed in Pubmed: 15869675.

11. Steckiewicz R, Górko D, Świętoń $E B$, et al. Axillary vein spasm during cardiac implantable electronic device implantation. Folia Morphol. 2016; 75(4): 543-549, doi: 10.5603/ FM.a2016.0027, indexed in Pubmed: 27830883.

12. Tse HF, Lau CP, Leung SK. A cephalic vein cutdown and venography technique to facilitate pacemaker and defibrillator lead implantation. Pacing Clin Electrophysiol. 2001; 24(4 Pt 1): 469-473, indexed in Pubmed: 11341084.

13. Wali MA, Eid RA, Dewan M, et al. Pre-existing histopathological changes in the cephalic vein of renal failure patients before arterio-venous fistula (AVF) construction. Ann Thorac Cardiovasc Surg. 2006; 12(5): 341-348, indexed in Pubmed: 17095976. 